\title{
BIBECHANA
}

A Multidisciplinary Journal of Science, Technology and Mathematics ISSN 2091-0762 (Print), 2382-5340 (Online)

Journal homepage: http://nepjol.info/index.php/BIBECHANA

Publisher: Research Council of Science and Technology, Biratnagar, Nepal

\section{Study of the stability of the perturbed solutions of the restricted three body problem}

\author{
M.A.A. Khan ${ }^{1}$, M.R. Hassan' ${ }^{2}$ R.R.Thapa ${ }^{3 *}$ \\ ${ }^{1}$ Teacher of Maths, Muslim Higher School, Bhagalpur-812002, India \\ ${ }^{2}$ Dept. of Mathematics, S.M. College, Bhagalpur, T.M.B. University, Bhagalpur - 812007, India \\ ${ }^{3}$ Dept. of Mathematics, P.G. Campus, Tribhuvan University, Biratnagar, Nepal \\ "E-mail: thaparajuram@yahoo.com
}

Article history: Received 14 August, 2015; Accepted 04 September, 2015

DOI: http://dx.doi.org/10.3126/bibechana.v13i0.13321

\section{Abstract}

In this paper we have been examined the stability of the perturbed solutions of the restricted three body problem. We have been restricted ourselves only to the first order variational equations. Our variational equations depend on the periodic solutions. Here the applications of the method of Fuchs and Floquet Proves to be complicated and hence we have been preferred Poincare's Method of determination of the characteristic exponents. With the determination of the characteristic exponents we have been abled to conclude regarding the stability of the generating solution. We have obtained that the motions are unstable in all the cases. By Poincare's implicit function theorem we have concluded that the stability would remain the same for small value of the parameter $\mu$ and in all types of motion of the restricted three-body problem.

CRCOST: All rights reserved.

Keywords: Characteristic equations; Characteristic exponents; Complex plane; Poincare's implicit function.

\section{Introduction}

According to poincare [1] there are three kinds of periodic solutions of the restricted three body problem. Planer case of three body problem depends on first and second kind solutions of the three body problem. Eccentricity is reduced to zero for solution of first kind but it does not reduced to zero for solution of second kind. Poincare observed the first kind solution in details. Kurcheeva [2] considered the second kind solution. Hassan et al. [3] also studied the effect of perturbation due to coriolis and centrifugal force on the periodic solution of the collision orbits of the restricted three body problem. 
In this paper we have examined the stability of the perturbed solutions of the restricted three body problem. For these characteristic equations of the periodic solutions are used to study.

\section{Characteristic equations of the periodic solutions}

The stability of a periodic solution depends on the variational system with periodic coefficients; and Poincare's characteristic equation become (Minorsky, [4])

$$
\left|\begin{array}{llll}
\frac{\partial \psi_{1}}{\partial \beta_{1}}+1-S & \frac{\partial \psi_{1}}{\partial \beta_{2}} & \frac{\partial \psi_{1}}{\partial \beta_{3}} & \frac{\partial \psi_{1}}{\partial \beta_{4}} \\
\frac{\partial \psi_{2}}{\partial \beta_{1}} & \frac{\partial \psi_{2}}{\partial \beta_{2}}+1-S & \frac{\partial \psi_{2}}{\partial \beta_{3}} & \frac{\partial \psi_{2}}{\partial \beta_{4}} \\
\frac{\partial \psi_{3}}{\partial \beta_{1}} & \frac{\partial \psi_{3}}{\partial \beta_{2}} & \frac{\partial \psi_{2}}{\partial \beta_{3}}+1-S & \frac{\partial \psi_{3}}{\partial \beta_{4}} \\
\frac{\partial \psi_{4}}{\partial \beta_{1}} & \frac{\partial \psi_{4}}{\partial \beta_{2}} & \frac{\partial \psi_{4}}{\partial \beta_{3}} & \frac{\partial \psi_{4}}{\partial \beta_{4}}+1-s
\end{array}\right|=0
$$

where, in our case

$$
\begin{aligned}
\psi_{i} & =x_{i}\left(s, \beta_{1}, \beta_{4}, \delta c, \mu\right)-x_{i}\left(o, \beta_{1}, \beta_{4}, \delta c, \mu\right), i=1,2,3,4 . \\
\mathrm{S}^{*} & =\frac{\pi \mathrm{K}}{4 \sqrt{\mathrm{C}}_{\mathrm{o}}}
\end{aligned}
$$

Substituting corresponding values in (1), we get $\frac{\partial \psi_{\mathrm{i}}}{\partial \beta_{\mathrm{j}}}$

$$
(S-1)^{2}\left|\begin{array}{ll}
A_{11}^{*}-S & A_{14}^{*} \\
A_{41}^{*} & A_{44}^{*}-S
\end{array}\right|=0
$$

Therefore, we get two values of $\mathrm{S}$ to be unity which implies that two characteristic exponents are zero; and

$$
S^{2}-S\left(A_{11}^{*}-A_{44}^{*}\right)-\left(A_{11}^{*} A_{44}^{*}-A_{14}^{*} A_{41}^{*}\right)=0
$$

If $|\mathrm{S}|<1$, then the solutions will be stable otherwise they will be unstable.

\section{Proof of stability of the solutions:}

Case I: $\left(\phi_{0}=0, \mathrm{w}= \pm \pi / 2\right)$

(i) $\quad$ K-even, m-odd, $\mathrm{S}^{*}=\frac{\pi \mathrm{K}}{4 \sqrt{\mathrm{C}}_{\mathrm{o}}}$ 


$$
\begin{aligned}
& \mathrm{A}_{11}^{*}= \pm(-1)^{(\mathrm{K}+\mathrm{m} \pm 1) / 2} \pi \mathrm{m} \\
& \mathrm{A}_{44}^{*}=-(-1)^{(\mathrm{K}+\mathrm{m} \pm 1) / 2} \pi \mathrm{m} \\
& \mathrm{A}_{14}^{*}=-(-1)^{(\mathrm{K}+\mathrm{m} \pm 1) / 2} \frac{\pi \mathrm{m}}{2 \mathrm{n} \sqrt{\mathrm{C}_{\mathrm{o}}}} \\
& \mathrm{A}_{41}^{*}=0
\end{aligned}
$$

Substituting these values in (3), we get

$$
\begin{aligned}
& \mathrm{S}^{2} \mp(-1)^{(\mathrm{K}+\mathrm{m} \pm 1) / 2} 2 \pi \mathrm{ms}+(-1)^{\mathrm{K}+\mathrm{m} \pm 1} \pi^{2} \mathrm{~m}^{2}=0 \\
& \Rightarrow \mathrm{S}= \pm(-1)^{(\mathrm{K}+\mathrm{m} \pm 1) / 2} \pi \mathrm{m} \\
& \quad= \pm \pi \mathrm{m}
\end{aligned}
$$

Let us change $S$ by $\frac{1+\lambda}{1-\lambda}$. This maps the interior of the unit circle onto the left half of the complex plane, so that the condition $/ S /<1$ is equivalent to $\operatorname{Re} \lambda<0$.

Applying this transformation, we get

$$
\lambda=\frac{\pi \mathrm{m}-1}{\pi \mathrm{m}+1} \text { or } \frac{\pi \mathrm{m}+1}{\pi \mathrm{m}-1}
$$

Which are positive as $\pi \mathrm{m}>1$. Therefore, the solutions are unstable.

(ii) K-odd, m-even, $\mathrm{S}^{*}=\frac{\pi \mathrm{K}}{4 \sqrt{\mathrm{C}}_{\mathrm{o}}}$

$$
\begin{aligned}
& \mathrm{A}_{11}^{*}=0, \mathrm{~A}_{44}^{*}=-(-1)^{(\mathrm{K}+\mathrm{m}+1) / 2} \frac{\pi \mathrm{m}}{\mathrm{n}} \\
& \mathrm{A}_{14}^{*}=-(-1)^{(\mathrm{K}+\mathrm{m}+1) / 2} \frac{\pi \mathrm{m}}{2 \sqrt{\mathrm{C}_{\mathrm{o}}}}, \mathrm{A}_{41}^{*}=-(-1)^{(\mathrm{K}+\mathrm{m}+1) / 2} 2 \sqrt{\mathrm{C}_{\mathrm{o}}} \pi
\end{aligned}
$$

Substituting these values in (3), we get

$$
\mathrm{S}^{2} \pm \mathrm{S} \frac{\pi \mathrm{m}}{\mathrm{n}}-\pi^{2} \mathrm{~m}^{2}=0
$$

Whose roots are real.

$$
\begin{aligned}
& \text { Applying } S=\frac{1+\lambda}{1-\lambda} \text { and taking +ve sign, we get } \\
& \lambda^{2}\left(\pi^{2} \mathrm{~m}^{2}-1+\pi \mathrm{m} / \mathrm{n}\right)-2\left(\pi^{2} \mathrm{~m}^{2}+1\right) \lambda+\left(\pi^{2} \mathrm{~m}^{2}-1-\pi \mathrm{m} / \mathrm{n}\right)=0 \\
& \therefore \lambda==\frac{\pi^{2} \mathrm{~m}^{2}+1 \pm \sqrt{ }\left\{4 \pi^{2} \mathrm{~m}^{2}+\left(\pi^{2} \mathrm{~m}^{2}\right) / \mathrm{n}^{2}\right\}}{\pi^{2} \mathrm{~m}^{2}-1+\pi \mathrm{m} / \mathrm{n}}=\frac{\pi^{2} \mathrm{~m}^{2}+1 \pm \pi \mathrm{m} \sqrt{\left(4+1 / \mathrm{n}^{2}\right)}}{\pi^{2} \mathrm{~m}^{2}-1+\pi \mathrm{m} / \mathrm{n}}
\end{aligned}
$$

Hence at least one value of $\lambda$ is positive as

$$
\pi^{2} \mathrm{~m}^{2}-1+\pi \mathrm{m} / \mathrm{n}=\pi \mathrm{m}^{2}(\pi+1 / \mathrm{K})-1>0, \quad(\therefore \mathrm{n}=\mathrm{K} / \mathrm{m})
$$

Therefore, the solutions are unstable.

And taking negative sign, we get

$$
\pi^{2}\left(\pi^{2} \mathrm{~m}^{2}-1-\frac{\pi \mathrm{m}}{\mathrm{n}}\right)-2 \lambda\left(\pi^{2} \mathrm{~m}^{2}+1\right)+\left(\pi^{2} \mathrm{~m}^{2}-1+\frac{\pi \mathrm{m}}{\mathrm{n}}\right)=0
$$




$$
\begin{aligned}
& \therefore \lambda=\frac{\pi^{2} \mathrm{~m}^{2}+1 \pm \sqrt{\left.\left\{4 \pi^{2} \mathrm{~m}^{2}+\pi^{2} \mathrm{~m}^{2}\right) / \mathrm{n}^{2}\right\}}}{\pi^{2} \mathrm{~m}^{2}-1-\pi \mathrm{m} / \mathrm{n}} \\
& =\pi \mathrm{m}^{2}(\pi-1 / \mathrm{K})-1>0 .
\end{aligned}
$$

Therefore, the solutions are unstable.

(iii) $\mathrm{K}$ - odd, $\mathrm{m}$ - odd, $\mathrm{S} * \frac{\pi \mathrm{K}}{4 \sqrt{\mathrm{C}_{\mathrm{o}}}}$

$$
\begin{aligned}
& \mathrm{A}_{11}^{*}=0, \mathrm{~A}_{44}^{*}=2 / \mathrm{n}(-1)^{(\mathrm{K}+\mathrm{m}) / 2}, \mathrm{~A}_{14}^{*}=-\frac{1}{2 \sqrt{\mathrm{C}}_{\mathrm{o}}}(-1)^{(\mathrm{K}+\mathrm{m}) / 2} \\
& \mathrm{~A}_{41}^{*}=-2 \sqrt{\mathrm{C}}_{\mathrm{o}}(-1)^{(\mathrm{K}+\mathrm{m}) / 2}
\end{aligned}
$$

Substituting these values in equation (3), we get

$$
n s^{2} \pm 2 s-n=0
$$

Whose roots are real.

$$
\begin{aligned}
& \text { Applying } S=\frac{1+\lambda}{1-\lambda}, \text { we get } \\
& \lambda^{2}+2 \mathrm{n} \lambda-1=0 \\
& \therefore \lambda= \pm \mathrm{n} \sqrt{\left(\mathrm{n}^{2}+1\right)= \pm\left\{\mathrm{n}+\sqrt{\left.\left(\mathrm{n}^{2}+1\right)\right\}}\right.}
\end{aligned}
$$

Therefore, one value of $\lambda$ is positive and consequently the solutions are unstable.

Case II: For $\phi_{0}=\pi / 2, \quad w=\pi i \quad(i=0,1,2, \ldots \ldots .$.

$$
\begin{aligned}
& \text { i) K-even, m-odd, } S^{*}=\frac{\pi \mathrm{K}}{4 \sqrt{\mathrm{C}_{\mathrm{o}}}} \\
& \mathrm{A}_{11}^{*}=-(-1)^{(\mathrm{K}+\mathrm{m}-1) / 2} \pi \mathrm{m}=\mathrm{A}_{44}^{*}, \mathrm{~A}_{14}^{*}=0, \mathrm{~A}_{41}^{*}=3 \mathrm{~L}_{41} \frac{\mathrm{t}_{0}^{*} \mathrm{p}_{0}^{*}}{\mathrm{n}}
\end{aligned}
$$

Substituting these values in equation (3), we get

$\left.\mathrm{S}^{2} \pm 2 \mathrm{~S}-1\right)^{(\mathrm{K}+\mathrm{m}-1) / 2} \pi \mathrm{m}+(-1)^{(\mathrm{K}+\mathrm{m}-1) / 2}=0$ and hence by case $\mathrm{I}(\mathrm{i})$, the solutions are unstable.

ii) K-odd, m-even, $\mathrm{S}^{*}=\frac{\pi \mathrm{K}}{4 \sqrt{\mathrm{C}}_{\mathrm{o}}}$

$$
\begin{aligned}
& \mathrm{A}_{11}^{*}=-(-1)^{(\mathrm{K}+\mathrm{m}-1) / 2} \pi \mathrm{m} / 2, \mathrm{~A}_{44}^{*}=0 \\
& \mathrm{~A}_{14}^{*}=(-1)^{(\mathrm{K}+\mathrm{m}-1) / 2} \frac{\pi \mathrm{m}}{2 \sqrt{\mathrm{C}}_{\mathrm{o}}}, \mathrm{A}_{41}^{*}=(-1)^{(\mathrm{K}+\mathrm{m}-1) / 2} 2 \pi \mathrm{m} \sqrt{\mathrm{C}_{\mathrm{o}}}
\end{aligned}
$$

Substituting these values in (3), we get

$\mathrm{S}^{2} \pm \frac{\pi \mathrm{m}}{\mathrm{n}} \mathrm{S}-\pi^{2} \mathrm{~m}^{2}=0$ and hence by case I (ii) the solutions are unstable.

iii) K-odd, m-odd, $\mathrm{S}^{*}=\frac{\pi \mathrm{K}}{4{\sqrt{\mathrm{C}_{\mathrm{o}}}}}$

$$
\begin{aligned}
& \mathrm{A}_{11}^{*}=-(-1)^{(\mathrm{K}+\mathrm{m}) / 2} \frac{2}{\mathrm{n}}, \mathrm{A}_{44}^{*}=0, \mathrm{~A}_{14}^{*}=-(-1)^{(\mathrm{K}+\mathrm{m}) / 2} \frac{1}{2 \sqrt{\mathrm{C}_{\mathrm{o}}}}, \\
& \mathrm{A}_{41}^{*}=-(-1)^{(\mathrm{K}+\mathrm{m}) / 2} 2 \sqrt{\mathrm{C}}_{\mathrm{o}}
\end{aligned}
$$

Substituting these values in (3), we get

$$
n \mathrm{~S}^{2} \pm 2 \mathrm{~S}-\mathrm{n}=0
$$


And hence by case I (iii), the solutions are unstable.

\section{Conclusion}

We have examined the stability of motion of the restricted three-body problem for the generating solutions applying direct method. We obtained that motions are unstable in all the cases. By virtue of Poincare's implicit function theorem we concluded that the stability would remain the same for small values of the parameters.

\section{References}

[1] Poincare, H. Los Method Nonvelles de la Machanique celeste, Gauthier-villars, Pairs, 1992. [2] I.V. Kurcheeve, Bull. Inst. of Theo. Astron. (1968)149.

[3] M. A. A. Khan, M. R. Hassan, R.R. Thapa, A research Journal in Mathematics, M $_{0}$ 1, 2 (2015) 48-58.

[4] N. Minorsky, Non-linear oscillations D. Van Nonstand Company, Inc. Toronto, New York, London, 1962. 